\title{
Do we use a priori knowledge of gravity when making elbow rotations?
}

\author{
Ilona J. Pinter $\cdot$ Arthur J. van Soest . \\ Maarten F. Bobbert · Jeroen B. J. Smeets
}

Received: 25 July 2011/Accepted: 6 December 2011/Published online: 29 December 2011

(C) The Author(s) 2011. This article is published with open access at Springerlink.com

\begin{abstract}
In this study, we aim to investigate whether motor commands, emanating from movement planning, are customized to movement orientation relative to gravity from the first trial on. Participants made fast point-to-point elbow flexions and extensions in the transverse plane. We compared movements that had been practiced in reclined orientation either against or with gravity with the same movement relative to the body axis made in the upright orientation (neutral compared to gravity). For each movement type, five rotations from reclined to upright orientation were made. For each rotation, we analyzed the first trial in upright orientation and the directly preceding trial in reclined orientation. Additionally, we analyzed the last five trials of a 30-trial block in upright position and compared these trials with the first trials in upright orientation. Although participants moved fast, gravitational torques were substantial. The change in body orientation affected movement planning: we found a decrease in peak angular velocity and a decrease in amplitude for the first trials made in the upright orientation, regardless of whether the previous movements in reclined orientation were made against or with gravity. We found that these decreases disappeared after participants familiarized themselves with moving in upright position in a 30-trial block. These results indicate that participants used a general strategy, corresponding to the strategy observed in situations with unreliable or limited information on external conditions. From this, we conclude that during movement planning, a priori
\end{abstract}

I. J. Pinter $(\square)$ - A. J. van Soest · M. F. Bobbert .

J. B. J. Smeets

Research Institute Move, Faculty of Human Movement Sciences,

VU University, Van der Boechorststraat 9, 1081 BT Amsterdam,

The Netherlands

e-mail: i.j.pinter@vu.nl knowledge of gravity was not used to specifically customize motor commands for the neutral gravity condition.

Keywords Motor planning - Gravity · Elbow rotations · Point-to-point movements

\section{Introduction}

In our daily life, we plan and make our movements in the constant presence of acceleration due to gravity. Gravity provides us with sensory information regarding our body orientation in the world (Carriot et al. 2008; Ebenholt 1970). However, it also interferes with the kinetics of our movements, because the gravitational torques that work on a body depend on the orientation of that body relative to gravity. This means that although the gravitational acceleration is constant, its effects on movement kinetics (gravitational effects) are not. Do we use the information with regard to our body orientation relative to gravity to predict these gravitational effects?

It has been shown by studying imagined movements that we know how gravity affects our movement execution (Papaxanthis et al. 2002). And although we have knowledge of the gravitational direction (Ebenholt 1970), it has been shown by Bringoux et al. (2004) that accuracy of this knowledge varied among tasks and was least accurate when the task implied making an arm movement. We aim to investigate whether a priori knowledge of gravitational effects is used to plan elbow movements. If it is, we would expect motor commands and movement kinematics to show that humans anticipate the gravitational effects. We use the term motor commands for all descending control signals coming from the supraspinal nervous system; those not only affect the states of alpha motoneuron activity but 
also influence the state of spinal reflex circuitry. Note that according to this definition, intrinsic viscoelastic muscle properties and muscle spindle feedback can (partly) compensate for a lack of correct anticipation of gravitational effects.

It has been shown that motor commands and kinematics vary with direction relative to gravity: Movements made against gravity show an early phasic antagonistic muscle activity that is not present in movements made with gravity (Virji-Babul et al. 1994). Movements made against gravity show a smaller time to peak angular velocity compared to movements made with gravity (Gentili et al. 2007; Papaxanthis et al. 1998). It has been argued that this difference in relative time to peak velocity for upward and downward movement is planned by the motor control system as this difference continues to exist (and only slowly disappears with practice) in a $0 \mathrm{~g}$ environment (Papaxanthis et al. 2005 Fig. 5 upper right panel). These ideas were confirmed by Le Seac'h and McIntyre (2007), who found that the smaller time to peak velocity for movements made against gravity compared to movements made with gravity remained when identical movements with respect to body axis are made in different body orientations relative to gravity. This substantiates that this difference in time to peak angular velocity arises from a different movement planning linked to the direction relative to gravity and not from biomechanical constraint linked to the direction relative to body axis.

The fact that the gravitational effects on a certain movement are anticipated after the same movement has been repeated several times does not imply that the brain can predict these gravitational effects when planning a first movement in a new gravity condition. It has been shown in force field experiments (Gribble and Ostry 2000; Thoroughman and Shadmehr 1999; Kistemaker et al. 2010; Kurtzer et al. 2005) that on a trial-to-trial basis, motor commands are customized to deal with external torques of various nature. This means we have to distinguish whether humans customize motor commands to gravity as they do for any external torque disturbance or whether they can predict how gravity will affect movement kinetics. Such a prediction would provide 'a priori' knowledge of the gravitational effects and enable motor commands emanating from movement planning to be customized to gravity from the first trial on.

In order to study how participants take into account a priori knowledge of gravity when performing arm movements, a setup was built in which the participants could be placed either in an upright orientation or in a reclined orientation. This allowed us to measure point-topoint elbow rotations that were identical with respect to the body axis, but occurred in different planes relative to gravity. The focus of this study will be on the first trials made in a new plane relative to gravity.
For these first trials in a new plane of movement we can formulate three possible outcomes, each based on a possible strategy for the use of a priori knowledge of the gravitational effects to customize motor commands. First, if participants do not use any a priori knowledge of the gravitational effects to customize motor commands to the new plane of movement (no customization strategy), kinematics will change in a way that can only be attributed to the manipulation of the gravitational effects. As it has been shown that motor commands are customized to the gravitational effects (Crevecoeur et al. 2009; Papaxanthis et al. 2005), we expect the kinematic changes found for the first trial in the new plane of movement to subside if successive trials in this plane are made.

As a second possible strategy, we would like to propose an aspecific customization of motor commands: a decrease in peak angular velocity and a decrease in amplitude independent of the manipulation of gravitational effects. We formulated the predictions for this strategy based on the findings that when confronted with uncertain external conditions participants tend to decrease movement speed and undershoot the target (Elliott et al. 2004; Hansen et al. 2003). During our experiment, participants are fully aware that they are placed in a different body orientation and will realize that the external conditions had changed. If they use this knowledge without knowing how the gravitational effects are influenced by the change in plane of movement, they might customize motor commands in an aspecific way by moving more carefully.

Furthermore, as motor commands are not specifically customized for the gravitational effects if an aspecific customization strategy is used, kinematics will also change as if no customization of motor commands occurs. And just as in the outcome predicted for the no customization strategy, the kinematic changes we find for the first trial in the new plane of movement should subside if successive trials in this plane are made.

As a third possible strategy, a priori knowledge of the gravitational effects is used to specifically customize motor commands to the gravitational effects (specific customization strategy) from the first trial on. In this case, contrary to the effects predicted for the first two strategies, any change in kinematics that we find for the first trial in the new plane of movement compared to the preceding trials will persist if successive trials in this plane are made.

\section{Method}

\section{Participants}

Twelve healthy participants ( 5 men and 7 women) with a mean age of 30 years (range 24-53 years) and without 
physical complaints at the shoulder, neck or arm participated in the experiment. Participants were included in the experiment when they succeeded in performing the experimental task both accurately (landing on a $4^{\circ}$ target area) and fast (at least $330 \%$ s) after at least 70 trials. One participant did not succeed and was excluded from the experiment. The local ethical committee approved the study. After receiving information about the experimental procedures, all participants signed an informed consent written in accordance with the declaration of Helsinki.

\section{Equipment}

Elbow angle was measured with a Spectrol 157 potentiometer (Vishay Electronic GmbH, Selb, Germany) installed in the rotational axis of a manipulandum. Angular acceleration at the elbow was derived from an ADXL321 accelerometer (Analog Devices, inc., Norwood, Massachusetts, United States) installed as a single-axis accelerometer at 0.4-m distance from the rotation axis of the manipulandum. All data were collected at a sample frequency of $1,000 \mathrm{~Hz}$ and synchronized with a pulse signal generated simultaneously with an audio signal that informed the participant to start a new movement.

\section{Experimental setup}

The participant was seated and fastened with safety belts in a seat that provided firm support of the trunk and scapula. The right lower arm was placed in a manipulandum positioned at shoulder height, which fixated the thoracohumeral angle at approximately $45^{\circ}$ adduction. A stationary lowfriction hinge aligned with the flexion/extension axis of the elbow only allowed elbow flexion and extension in the transversal plane of the participant. In upright orientation, elbow rotations were performed in the horizontal plane (neutral gravity). The complete setup could be tilted backward by $70^{\circ}$ (reclined orientation). In this orientation, elbow rotations were performed in a nearly vertical plane, either against or with gravity (see Fig. 1). For the remainder of this article, we will refer to this orientation as 'moving in the vertical plane'. The additional mass of the manipulandum placed the position of the center of mass of lower arm and manipulandum together (CoM) laterally of the lower arm; the mean (SD) angular deviation between the longitudinal axis of the lower arm and the line between the CoM and the axis of the low-friction hinge was $14(4)^{\circ}$ (see Appendix A). Mean (SD) inertia (of the lower arm and the manipulandum together) relative to the elbow joint was $0.111(0.009) \mathrm{kgm}^{2}$ (see Appendix B).

Participants performed fast elbow rotations from one target to another. Three visual targets, with a width of $4^{\circ}$ and a center-to-center distance of $35^{\circ}$, were present with

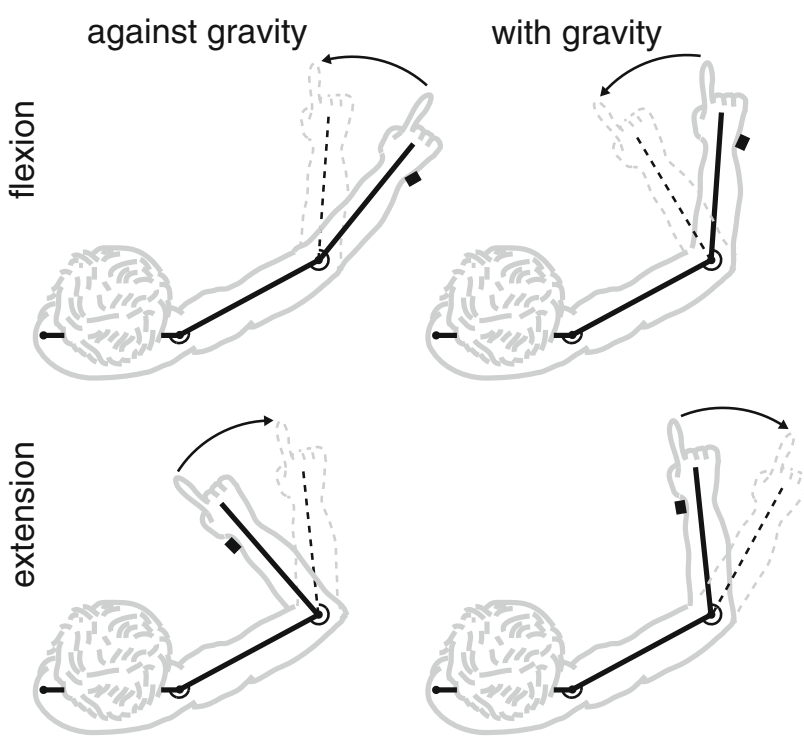

Fig. 1 The four movement types that were used as experimental task. Movements are defined based on their body-related movement direction and direction relative to gravity when moving in vertical plane (reclined orientation)

the middle target chosen such that when pointing at this target in a reclined orientation, the gravitational torque working on the center of mass of lower arm and manipulandum together was close to zero. By this choice of targets, elbow flexions and elbow extensions made in the vertical plane were either made against gravity or with gravity with a total of four movement types (see Fig. 1): 2 anatomical directions $\times 2$ gravitational directions. When placed in upright orientation, elbow rotations that were identical with respect to the body axis were made in the horizontal plane of movement.

When moving in the vertical plane, the lower arm of the participants was supported, while they were waiting for the starting signal. This was done to postpone muscle fatigue and to provide a similar muscle state at the beginning of each trial regardless of whether the movement to be made was in the vertical or horizontal plane. This justified the assumption of similar motor commands in our no customization strategy. Additionally, the arm support accommodated anti-gravitational torques before the start of each trial. This means that proprioceptive information on gravitational load (which we regard as a posteriori knowledge) was not available. For making movements against gravity, the net muscle torque first had to compensate for the gravitational torques before acceleration occured. For each movement type, participants practiced for at least 70 trials before measurements started. This gave them ample opportunity to learn gravitational load and to customize motor commands.

A wooden board blocked vision of the moving arm. Once participants had finished the movement, they were 
given knowledge of results by means of a laser shining from the longitudinal axis of the lower arm to the target. This knowledge of results was given to prevent drift in the visuomotor calibration (Smeets et al. 2006).

In addition, participants were given feedback on their movement speed by an auditory signal; they were told "Come on!" if they had moved with a peak angular velocity that fell below a preset threshold. This threshold was set for each participant separately (mean (SD): $\left.370(22)^{\circ} / \mathrm{s}\right)$ in the first practice block but was never set below $330^{\circ} / \mathrm{s}$. The threshold was set such that participants had to make an effort to be fast enough and were still successful in the majority of trials.

\section{Experimental procedure}

A short pretest was done to calculate the position of the center of mass of the lower arm and manipulandum together (see Appendix A). Additionally, a quick-release experiment was done to calculate moment of inertia of the lower arm and manipulandum together (see Appendix B). The parameters derived from these measurements were used to calculate the gravitational torques and the net muscular torques from the angular position and angular acceleration data (see Appendices A and B). Before starting the main measurements, a 5-second measurement was done to obtain the elbow angle when participants pointed accurately at the center of the target. This target-elbow angle calibration was used to determine how well movements ended on the target.

Since moving in an upright orientation is common in daily life situations, we assume that an internal model of gravity should be well adapted to this body orientation. This was taken into account in the experimental design. Participants first practiced in the less common reclined orientation (vertical plane of movement) after which they were placed in upright orientation (horizontal plane of movement). We analyzed the first trial in the horizontal plane of movement to see whether motor commands were customized to the gravitational effects that were different from the preceding trials (but otherwise very common).

The four movement types (see Fig. 1) were performed in four separate blocks. The order in which blocks were presented was counterbalanced. Before starting a new block, participants practiced 70 trials in the vertical plane to familiarize themselves with the gravitational effects, the additional mass of the manipulandum and the resulting shift of the position of the center of mass. It has been shown that on average, participants need between 64 and 128 trials to fully customize motor commands to cope with external forces (Thoroughman and Shadmehr 1999). In the interest of keeping total duration of the measurements within $3 \mathrm{~h}$ for each participant, we choose a practice block

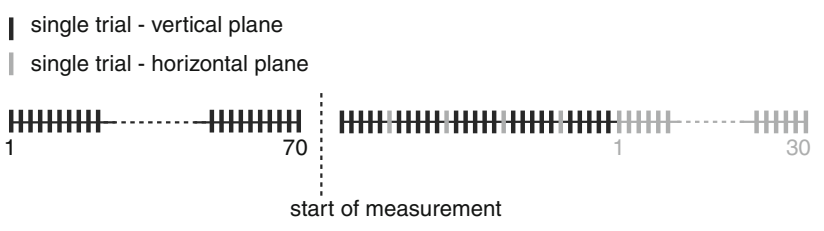

Fig. 2 Schematic representation of the time line of the trials. After a practice session of 70 trials in the vertical plane (reclined position), the measurement started (see vertical dashed line). Five trials of moving in the vertical plane were alternated with one trial in the horizontal plane (upright position). After the fifth alteration, the one trial in horizontal plane was followed by an additional 29 trials

of 70 trials. After the practice block, the measurements started. Five trials in the vertical plane were followed by one trial in the horizontal plane. This was repeated five times, followed directly by 29 trials in horizontal plane during which participants could customize motor commands to moving in the horizontal plane. For an overview of the trials see Fig. 2. To prevent exploration of gravity before starting the first trial in horizontal plane, participants were instructed to keep their arm at the starting position when the setup was tilted upward and wait for the signal to start a new movement. Before the start of each trial, participants were asked to point at the target indicated as starting point and wait for the auditory starting signal, subsequently look at the target indicated as the goal of the movement and make a single movement from the starting target to the goal target as fast as possible.

\section{Data processing and statistics}

The position signal was filtered bidirectionally with a second-order Butterworth filter (cutoff $30 \mathrm{~Hz}$ ) and differentiated to obtain a velocity signal. The peak angular velocity $\left(\omega_{\text {peak }}\right)$ was calculated as a measure of movement velocity. The point where the movement came to a standstill was defined as the first instant after reaching peak angular velocity at which absolute angular velocity dropped below $5 \%$ and stayed below $5 \%$ for at least $100 \mathrm{~ms}$. We took the difference between the elbow angle at standstill and the elbow angle when pointing at the center of the target $(\Delta \theta$, overshoot) as a measure of how participants had planned to end their first movement based on open loop motor commands. Although participants had enough time to adjust motor commands during the movement, our experimental task was designed not to provide direct information on task performance before movement came to a standstill. Because information on initial posture, target location and end position of the movement was all provided visually, we may assume that supraspinal feedback to adapt motor commands based on movement error was primarily based on visual information (Pipereit et al. 2006; Sober and Sabes 2003). Since visual information was not 
available until the end of the movement, we assume that motor commands were not changed during an ongoing movement. In addition to the kinematic parameters $\Delta \theta$ and $\omega_{\text {peak }}$, we also calculated the relative time to peak angular velocity using the methodology as described by Crevecoeur et al. (2009). We will use this measure to describe how our experimental results relate to previous published research.

For each participant and each movement type (see Fig. 1), five switches from the vertical to the horizontal plane were made. This results in five first trials in the horizontal plane that we analyzed (condition $\mathrm{HP}_{\text {unadapt }}$ ). Kinematic parameters of these five $\mathrm{HP}_{\text {unadapt }}$ trials were compared with those of the trials directly preceding them in the vertical plane (condition $\mathrm{VP}_{\text {adapt }}$ ). In addition, the last five trials (condition $\mathrm{HP}_{\text {adapt }}$ ) from the final 30-trial block in the horizontal plane were analyzed and compared with trials in $\mathrm{HP}_{\text {unadapt }}$ condition.

The kinematic parameters $\Delta \theta$ and $\omega_{\text {peak }}$ were averaged over the five trials obtained for each of the three conditions $\left(\mathrm{VP}_{\text {adapt }}, \mathrm{HP}_{\text {unadapt }}\right.$ and $\mathrm{HP}_{\text {adapt }}$ ) and each of the four movement types. A 2 (anatomical direction) $\times 2$ (gravitational direction) $\times 3$ (condition) repeated measures ANOVA was done for both variables to identify which of the three possible strategies as stated in the introduction could be rejected by our data. According to the no customization strategy, the change in orientation will affect $\Delta \theta$ and $\omega_{\text {peak }}$, and this effect will depend on the gravitational effects encountered in the preceding trials. If in the preceding trials the movements had been made against gravity, we expect movement velocity to increase and the movement endpoint to fall beyond the target position. If in the preceding trials movements had been made with gravity, we expect movement velocity to decrease and movement endpoint to fall before target position. This means that we will accept the no customization strategy if a repeated measures ANOVA will show an interaction effect of (gravitational direction) $\times$ (condition), indicating that $\Delta \theta$ and $\omega_{\text {peak }}$ decreased if in preceding trials movement was made with gravity and $\Delta \theta$ and $\omega_{\text {peak }}$ increased if in preceding trials movement was made against gravity. Since we predict that this effect would show for the first trial in horizontal plane and subside when successive trials in the horizontal plane are made, we used planned contrasts to see whether this interaction effect was significant for the $\mathrm{HP}_{\text {unadapt }}$ compared to the $\mathrm{VP}_{\text {adapt }}$ and the $\mathrm{HP}_{\text {adapt }}$.

If the aspecific customization strategy is used (participants simply move more careful), the first trial in horizontal plane will show a decrease in the movement speed and a decrease in overshoot compared to movements made in vertical plane no matter whether the preceding movements in vertical plane were made against or with gravity. We will accept this aspecific customization strategy if our repeated measures ANOVA will show a main effect of condition, indicating that $\omega_{\text {peak }}$ and $\Delta \theta$ decreased for the first trial in the horizontal plane. Since we predict this decrease to subside if successive trials in the horizontal plane are made, we again used planned contrasts to see whether the main effect is significant for the $\mathrm{HP}_{\text {unadapt }}$ compared to the $\mathrm{VP}_{\mathrm{adap}}$ and the $\mathrm{HP}_{\text {adapt }}$. We expect for this strategy that motor commands are not specifically customized to the gravitational effects in the horizontal plane from the first trial on. Therefore, in addition to the previously mentioned main effect, we might also find that for the first trial in the horizontal plane, kinematics are affected differently when in preceding trials movements were made with gravity compared to when in preceding trial movements were made against gravity. This will result in the same interaction effect as predicted for the no customization strategy.

We will accept the specific customization strategy if we find either no main effect on condition in our repeated measures ANOVA or a main effect on condition with planned contrasts indicating a difference for $\mathrm{HP}_{\text {unadapt }}$ compared to $\mathrm{VP}_{\text {adapt }}$ but not for $\mathrm{HP}_{\text {adapt }}$ compared to $\mathrm{HP}_{\text {unadapt }}$.

\section{Results}

Figure 3 shows results of elbow flexions in $\mathrm{VP}_{\text {adapt }}$, $\mathrm{HP}_{\text {unadapt }}$ and $\mathrm{HP}_{\text {adapt }}$ conditions. As can be seen in the bottom plot, the gravitational torques were substantial compared to the net muscular torques. The maximal gravitational torques during movement were approximately $19 \%$ of the maximal net muscular torque. For the $\mathrm{VP}_{\mathrm{adap}}$ condition, we found a mean (intersubject standard error of the mean) relative time to peak angular velocity of $0.540 .01)$ for movements made against gravity and 0.49 (0.01) for movements with gravity. This difference between movements made with gravity compared to movements made against gravity remained 5\% when identical movements relative to body axis were made in the horizontal plane ( $\left.\mathrm{HP}_{\text {adapt }}\right): 0.52(0.01)$ and $0.47(0.01)$ consecutively.

Figure 4 shows the mean values for $\Delta \theta$ and $\omega_{\text {peak }}$ over all participants for movements made in the $\mathrm{VP}_{\text {adapt }}, \mathrm{HP}_{\text {unadapt }}$ and $\mathrm{HP}_{\text {adapt }}$ conditions. Results of the 2 (anatomical direction) $\times 2$ (gravitational direction) $\times 3$ (condition) repeated measures ANOVA are shown in Table 1 . We found a significant main effect on anatomical direction for $\omega_{\text {peak }}$, indicating that overall elbow extensions had a lower $\omega_{\text {peak }}$ than elbow flexions. This difference was only present in the movements made with gravity and not in movements made against gravity indicated by a significant interaction effect (anatomical direction) $\times$ (gravitational direction) for $\omega_{\text {peak }}$. We also found a significant main effect on gravitational direction for $\Delta \theta$. As clearly shown in Fig. $4, \Delta \theta$ was smaller 

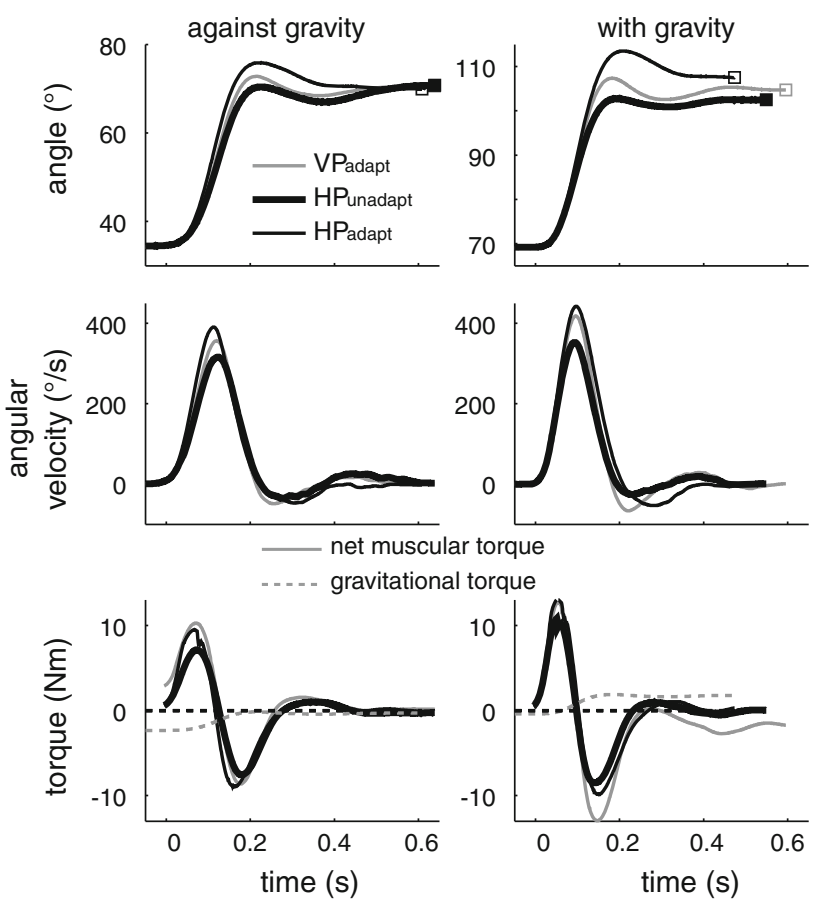

Fig. 3 An example of position ( $0^{\circ}$ indicating full elbow extension), velocity and net muscular torque data averaged over the five trials made either against or with gravity in de vertical plane $\left(\mathrm{VP}_{\text {adapt }}\right)$, the five first trials made in horizontal plane ( $\mathrm{HP}_{\text {unadapt }}$ ) and the last five trials in horizontal plane $\left(\mathrm{HP}_{\text {adapt }}\right)$. The squares indicate the point where movement came to a standstill. Data of elbow flexions made by participant 11 were used. The gravitational torque (dashed lines) encountered when moving in the vertical plane was substantial compared to the net muscular torque (solid lines)

for movements made with gravity than for movement made against gravity.

The main effect on condition and the interaction effect (gravitational direction) $\times$ (condition) are relevant to identify which customization strategy was used. If one of these two effects was significant, a planned contrast was done (see Table 2). We found that for these two effects, there was no difference for elbow flexions and extensions (Table 1: no interaction effect (anatomical direction) $\times$ (condition) and no interaction effect (anatomical direction) $\times$ (gravitational direction $) \times($ condition $))$. This justifies that in Fig. 4 we simplified our data by averaging the elbow flexions en elbow extensions for the movements made with gravity and for the movements made against gravity.

We reject the no customization strategy because we found no interaction effect of (gravitational direction) $\times($ condition $)$ for $\omega_{\text {peak }}$. Had participants ignored gravity altogether, one would expect peak angular velocity and overshoot in the horizontal plane to increase when in preceding trials the movements were made against gravity and decrease when in preceding trials movements were made with gravity. Instead, we observed that these parameters decreased regardless of the previous movement

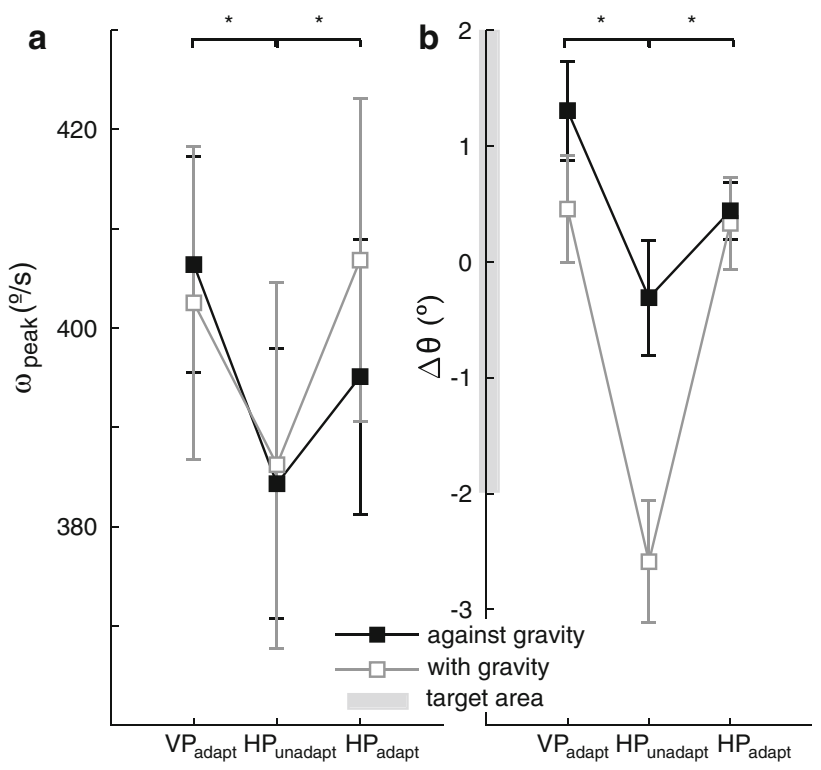

Fig. 4 The kinematic parameters peak angular velocity $\left(\omega_{\text {peak }}\right)$ and overshoot $(\Delta \theta)$ averaged over all twelve participants. The error bars indicate the intersubject standard error of the mean. The data for elbow flexions and extensions were averaged, resulting in one set of (open gray) symbols for movements made with gravity and one set of (filled black) symbols for movements made against gravity. Significant differences between conditions as found in the planned contrasts are indicated with asterisk. Both in movements made against and in movements made with gravity, peak angular velocity and overshoot decreased for the first trial made in horizontal plane $\left(\mathrm{HP}_{\text {unadapt }}\right)$ compared to the preceding trials made in the vertical plane $\left(\mathrm{VP}_{\text {adapt }}\right)$ and the following trials in the horizontal plane $\left(\mathrm{HP}_{\text {adapt }}\right)$. The gray bar indicates the target area

direction. This means that participants changed motor commands. We also reject the specific customization strategy because we found a main effect on condition with $\mathrm{HP}_{\text {adapt }}$ differing from $\mathrm{HP}_{\text {unadapt }}$. Thus, participants customized their motor commands on a trial-to-trial basis when successive trials in the horizontal plane were made. For the main effect on condition, we also found that $\mathrm{HP}_{\text {unadapt }}$ differed from $\mathrm{VP}_{\text {adapt }}$. In summary, we found a decrease in $\omega_{\text {peak }}$ and $\Delta \theta$ when participants moved in the horizontal plane for the first time and an increase in $\omega_{\text {peak }}$ and $\Delta \theta$ when successive trials in the horizontal plane were made (see Fig. 4). These results indicate that the data are in line with the predictions made for aspecific customization strategy of motor commands.

Additionally, we found a significant interaction effect (gravitational direction) $\times($ condition $)$ for $\Delta \theta$ : The decrease in $\Delta \theta$ for $\mathrm{HP}_{\text {unadapt }}$ compared to $\mathrm{VP}_{\text {adapt }}$ and $\mathrm{HP}_{\text {adapt }}$ was stronger when preceding movements were made with gravity compared to when preceding movements were made against gravity (see Fig. 4). This indicates that the effects we found for the first trial in the horizontal plane depended on the gravitational effects encountered in the preceding trials and that as participants 
Table 1 Results from the 2 (anatomical direction) $\times 2$ (gravitational direction) $\times 3$ (condition) repeated measures ANOVA for $\Delta \theta$ and $\omega_{\text {peak }}$

\begin{tabular}{|c|c|c|}
\hline & $\omega_{\text {peak }}$ & $\Delta \theta$ \\
\hline \multicolumn{3}{|l|}{ Main effects } \\
\hline (anatomical direction) & $F_{2,11}=5.258, P=0.043$ & - \\
\hline (gravitational direction) & - & $F_{2,11}=14.746, P=0.003$ \\
\hline$(\text { condition })^{\#}$ & $F_{2,11}=8.471, P=0.002$ & $F_{2,11}=38.696, P \ll 0.001$ \\
\hline \multicolumn{3}{|l|}{ Interaction effects } \\
\hline (anatomical direction $) \times($ gravitational direction $)$ & $F_{1,22}=13.108, P=0.004$ & - \\
\hline$($ gravitational direction $) \times(\text { condition })^{\#}$ & - & $F_{1,22}=9.735, P=0.001$ \\
\hline (anatomical direction $) \times($ condition $)$ & - & - \\
\hline$($ anatomical direction $) \times($ gravitational direction $) \times($ condition $)$ & - & - \\
\hline
\end{tabular}

The effects indicated with ${ }^{\#}$ are relevant to indentify which customization strategy was used. For these effects, planned contrasts were done if they showed to be significant (Table 2)

Table 2 Planned contrast for the main effect on condition and the significant interaction effects (gravitational direction) $\times($ condition)

\begin{tabular}{lll}
\hline & $\omega_{\text {peak }}$ & $\Delta \theta$ \\
\hline Main effects condition & & $F_{1,11}=53.122, P \ll 0.001$ \\
$\mathrm{HP}_{\text {unadapt }}$ versus $\mathrm{VP}_{\text {adapt }}$ & $F_{1,11}=17.785, P=0.001$ & $F_{1,11}=52.362, P \ll 0.001$ \\
$\mathrm{HP}_{\text {adapt }}$ versus $\mathrm{HP}_{\text {unadapt }}$ & $F_{1,11}=6.964, P=0.023$ & \\
Interaction effects (gravitational direction) $\times($ condition) & - & $F_{1,11}=13.503, P=0.004$ \\
$\mathrm{HP}_{\text {unadapt }}$ versus $\mathrm{VP}_{\text {adapt }}$ & - & $F_{1,11}=13.898, P=0.003$ \\
$\mathrm{HP}_{\text {adapt }}$ versus $\mathrm{HP}_{\text {unadapt }}$ & - & \\
\hline
\end{tabular}

The first trials in the horizontal plane $\left(\mathrm{HP}_{\text {unadapt }}\right)$ was compared to the preceding trials in the vertical plane $\left(\mathrm{VP}_{\text {adapt }}\right)$ and the last trials in the horizontal plane $\left(\mathrm{HP}_{\text {adapt }}\right)$ was compared to the first trials in the horizontal plane $\left(\mathrm{HP}_{\text {unadapt }}\right)$ are shown

planned to undershoot the target, motor commands were not specifically customized to the new gravitational effects.

We found a substantial difference in movement parameters between $\mathrm{HP}_{\text {unadapt }}$ and $\mathrm{HP}_{\text {adapt }}$. In order to determine how fast the movements adapt to the changed gravity condition, we plotted the trial-by-trial adaptation for the final 30-trial block in the horizontal plane (Fig. 5). The last five trials of this block constitute the $\mathrm{HP}_{\text {adapt }}$ condition; the first trial of this block is (together with the first trial of the short blocks) the $\mathrm{HP}_{\text {unadap }}$ condition. This figure clearly shows that the adaptation to the horizontal plane occurs for a large extent immediately after the first trial and can be regarded as one-trial learning.

\section{Discussion and conclusions}

We compared fast and accurate point-to-point elbow rotations that had been practiced in the vertical plane with identical movements with respect to body axis made in the horizontal plane. For the first trials in the horizontal plane, movement kinematics changed: Both peak angular velocity and the overshoot reduced regardless of whether the previous movements were made against or with gravity. These results contradict the no customization strategy. We furthermore found that the decrease in both the peak angular velocity and the overshoot disappeared after participants familiarized themselves with moving in the horizontal plane. These results contradict the specific customization strategy. We conclude that for the first trial, a new plane of movement relative to gravity, an aspecific customization strategy is used, suggesting that a priori knowledge of the gravitational effects either is limited or unreliable.

Is a priori knowledge of the gravitational effects specific?

For each participant and each movement, five switches were made from moving in the vertical plane to moving in the horizontal plane. We analyzed all first trials after this switch as if participants were still naïve to moving in the horizontal plane. Apparently they were, otherwise we would not have found that aspecific customization of motor commands takes place in the first trial.

We found that for the first trial in a new plane of movement relative to gravity, motor commands were a specifically customized: Participants planned to move slower and to reduce overshoot when confronted with a new plane of movement. This conservative strategy has 


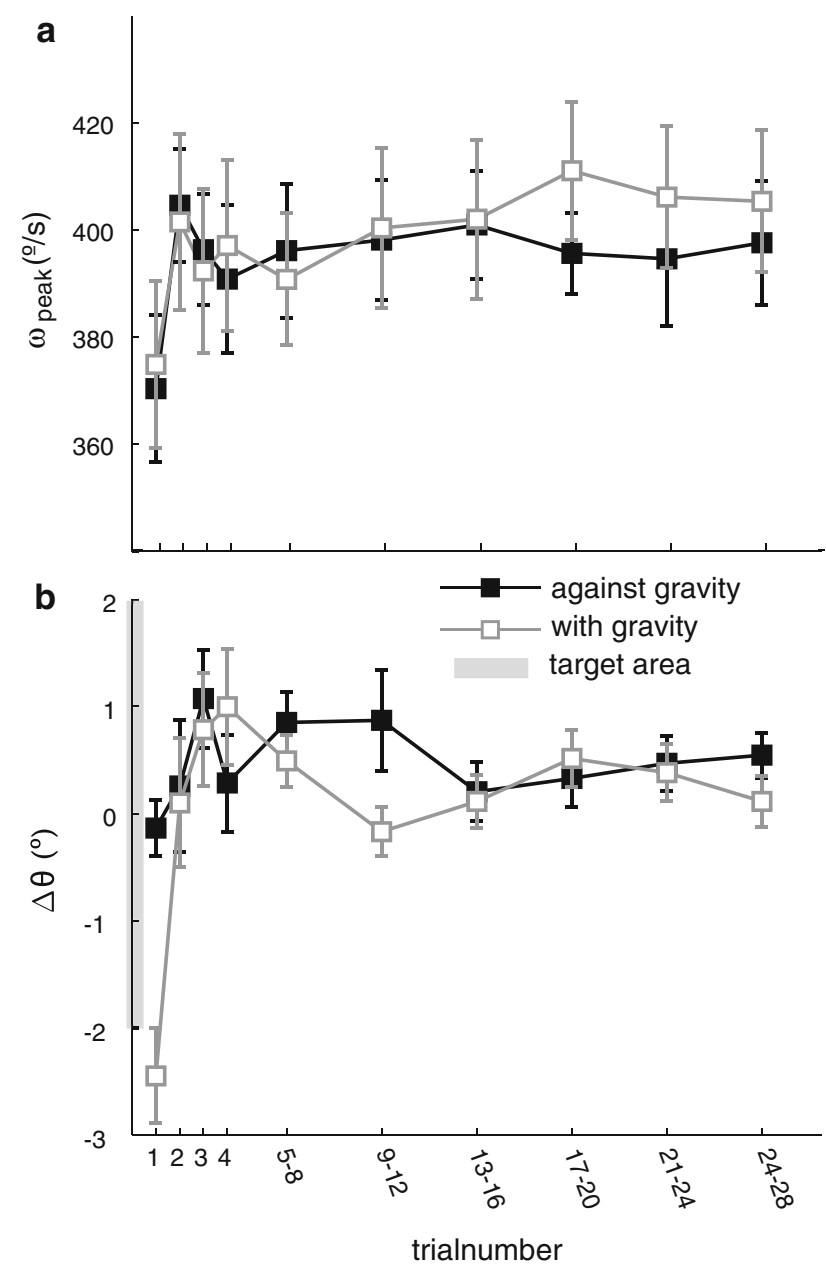

Fig. 5 The trial-by-trial adaptation of the peak angular velocity $\left(\omega_{\text {peak }}\right)$ and the overshoot $(\Delta \theta)$ as a function of trial number after the switch to the horizontal plane. The mean over all twelve participants is shown, and the error bars indicate the standard error of the mean

been reported in experiments in which participants were asked to move both fast and accurately and were confronted with external conditions for which information was limited (Elliott et al. 2004; Hansen et al. 2003). If participants used this conservative strategy because a priori knowledge of the gravitational effects for the new plane of movement was limited, we expect kinematics to show that motor commands are not yet specifically customized to the gravitational effects for the horizontal plane and anticipate the gravitational effects encountered in the preceding trials in the vertical plane. In line with this reasoning, we found a significantly stronger decrease in overshoot for the first trial in the horizontal plane after following movements made with gravity compared to movements made against gravity (the gray lines are steeper than the black lines in Fig. 4b).

We can quantify the effects predicted in the previous paragraph. In the movements made with gravity, we can estimate that the gravitational torque at the planned endpoint is approximately $2.3 \mathrm{Nm}$ (Eq. A.1). When moving in the vertical plane, motor commands need to result in a net joint torque that exactly compensates this gravitational torque in the planned endpoint. If on the first trial in horizontal plane motor commands are not specifically customized, movement will not end at the target but fall short. Based on experimentally obtained values for elbow stiffness, we can estimate how much participants would fall short. At a static elbow stiffness of $38 \mathrm{Nm} / \mathrm{rad}$ (MussaIvaldi et al. 1985, Table 1: mean value over all postures), we predict that movement would fall short by $2.3 / 38 \mathrm{rad}$ or $3.5^{\circ}$. We found that overshoot decreased by $3.0^{\circ}$ for the first trial in the horizontal plane compared to the last trial in the vertical plane, which is nicely in line with the prediction. We conclude that for the first trial in the horizontal plane, motor commands were still set to oppose a gravitational torque at the endpoint as encountered when moving in the vertical plan.

We may assume that knowledge of the body orientation relative to gravity was available to the participants $(\mathrm{Eb}-$ enholt 1970; Bringoux et al. 2004), based on interoception (for instance the vestibular system), vision and contact forces between the chair and the body (Carriot et al. 2008; Lackner and DiZio 2000). Since we find that participants used an aspecific customization strategy, we may conclude that participants were indeed aware that external conditions had changed when switching from moving in the vertical plane to moving in the horizontal plane. Our results show that this knowledge was not used for a specific customization of motor commands. This suggests that participants did not have specific a priori knowledge of gravitational effects but only realized that orientation relative to gravity changed.

Previous published research on movement planning and gravity shows a difference in planned movement kinematics with respect to movement direction relative to gravity. Movements made against gravity are planned with a smaller relative time to peak velocity than movements made with gravity (Papaxanthis et al. 2005; Le Seac'h and McIntyre 2007). The movements in the present study do not show this difference. The small difference in relative time to peak angular velocity we found for moving with compared to moving against gravity was opposite compared to previous studies (Papaxanthis et al. 2005), but the same difference was also present without gravity (when moving in the horizontal plane). We attribute the lack of effect on time to peak angular velocity in our experiment to the fact that our participants were asked to move as fast as possible. As the duration of acceleration and deceleration periods was already minimal, participants had no room to shift the relative time to peak velocity without compromising the task performance of moving maximally fast. 
We deliberately used maximally fast movements to be able to investigate how movements are planned when only a priori (and no online) knowledge of gravitational effect is available. This is a completely different approach compared to previous published research in which a posteriori knowledge on the gravitational effects was available from previous trials or from proprioceptive information by holding the arm in the starting position without an arm support (Crevecoeur et al. 2009; Gentili et al. 2007; Le Seac'h and McIntyre 2007; Papaxanthis et al. 2005; VirjiBabul et al. 1994, etc.). With this innovative approach, we show that for the first trial in a new plane of movement when only a priori knowledge of the gravitational effects is available, the planning of elbow movement was dominated by a conservative strategy leading to an aspecific customization of motor commands. This strategy is directly abandoned when a posteriori knowledge of the gravitational effects is available: From the second trial on motor, commands are specifically customized to the gravitational effects (see Fig. 5), and overshoot is restored to values (Fig. 4) similar to those found when movements were made in the vertical plane. Such a fast correction of endpoint error might seem surprising, but one-trial learning combined with systematic but small fluctuations after the second trial (Fig. 5) seems very much in line with the theory of optimal feedback control (Scott 2004; Todorov and Jordan 2002).

With the methodology used in the present study, we found that a priori knowledge of gravitational effects is used to aspecifically customize motor commands when planning elbow rotations. This reduces the contribution of an a priori internal model of gravity to take into account body orientation when planning elbow movements. For future research, we suggest that the use of a priori knowledge of gravitational effects is investigated in other movements. This will contribute to the general ideas on how the brain predicts the gravitational effects on a planned movement.

Open Access This article is distributed under the terms of the Creative Commons Attribution Noncommercial License which permits any noncommercial use, distribution, and reproduction in any medium, provided the original author(s) and source are credited.

\section{Appendix A}

Since the setup only allowed elbow flexion and extension in the transverse plane, we will describe all equations of motion in the two degrees-of-freedom of this plane of motion. The orientation of the plane of motion with respect to gravity can be described by $\varphi_{\text {reclined }}$ (see Fig. 4a). For all participants $\varphi_{\text {reclined }}$ was either $0^{\circ}$ or $70^{\circ}$.

The gravitational torques relative to the rotational axis of the low-friction hinge $(R)$ encountered during the movement were derived from the elbow angle data using equation:

$T_{\text {grav }}=g \cdot m \cdot d \cdot \cos \left(\varphi_{\mathrm{CoM}}\right) \cdot \sin \left(\varphi_{\text {reclined }}\right)$

in which $T_{\text {grav }}$ is the gravitational torque, $g$ the acceleration due to gravity, $m$ the mass of the lower arm and manipulandum together, $d$ the distance from the center of mass of lower arm and manipulandum together (CoM) to $R$ and $\varphi_{\mathrm{CoM}}$ the angle between the line connecting $R$ and CoM and the line $r$ (see Fig. 4b).

Within the plane of movement, $\varphi_{\mathrm{CoM}}$ can be expressed in terms of the elbow angle $\left(\varphi_{\mathrm{elb}}\right)$, which was measured during the experiments, and the shoulder angle $\left(\varphi_{\mathrm{sh}}\right)$, which was fixed at $30^{\circ}$, and a constant $\Delta \varphi_{\mathrm{CoM}}$ (see Fig. 6b):

$\varphi_{\mathrm{CoM}}=\varphi_{\mathrm{sh}}+\varphi_{\mathrm{elb}}+\Delta \varphi_{\mathrm{CoM}}$

The parameters $m \cdot d$ and $\Delta \varphi_{\mathrm{CoM}}$ were estimated for each participant separately as described in the following paragraph.
Fig. 6 The setup used to estimate the position of the center of mass and the mass of lower arm and manipulandum together. a The setup was tilted backward, so that the plane of motion was nearly vertical. Orientation of the plane of motion relative to gravity can be described by $\varphi_{\text {reclined }}$. b Top view in body-related coordinate system is shown. The lower arm and manipulandum (shown in black) are attached to a force transducer. Angle definitions are shown

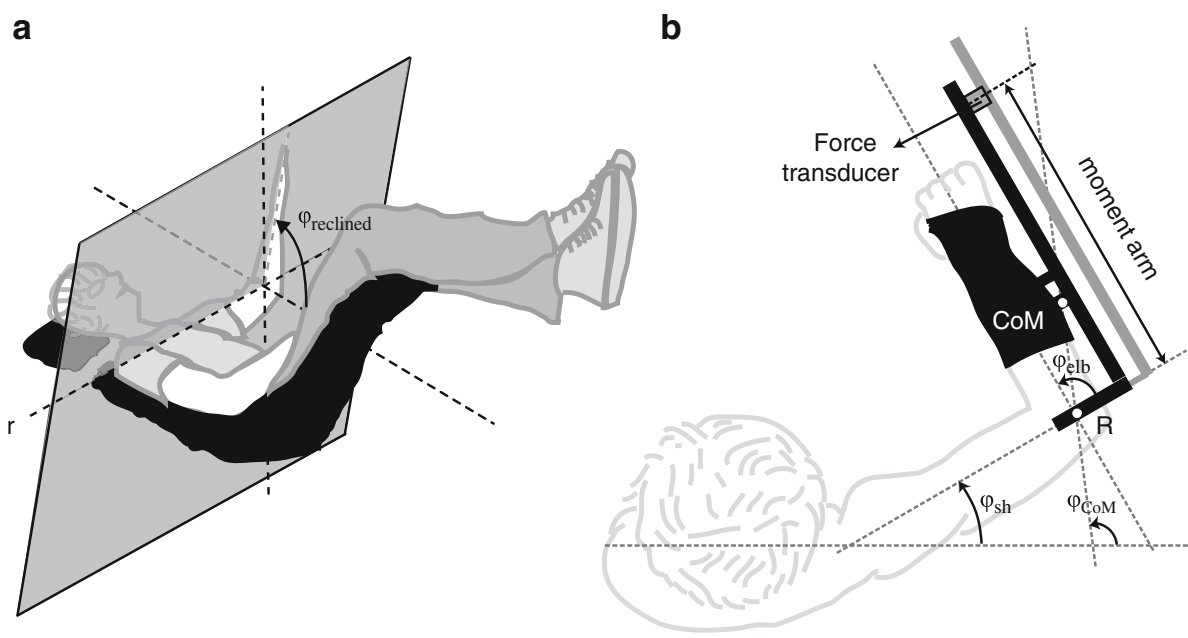


First, the lower arm and manipulandum together were attached to a KAP-E $(2,000 \mathrm{~N})$ force transducer (AST Mess and Regeltechnik, Dresden, Germany) at a $0.3-\mathrm{m}$ distance of the rotation axis $R$, and the setup was placed in the $70^{\circ}$ reclined orientation. The participant was instructed to relax the arm and shoulder muscles. In a short static measurement, we determined the elbow angle and gravitational torque relative to $R$. This measurement was repeated at various elbow angles to obtain at least three calibration points for the equation:

$T_{\text {grav }}=a \cdot \sin \left(\varphi_{\mathrm{sh}}+\varphi_{\mathrm{elb}}+\Delta \varphi_{\mathrm{CoM}}\right)+b$

The parameter $\Delta \varphi_{\mathrm{CoM}}$ was optimized by finding a least squared difference solution for $a$ and $b$ with the additional constrained to keep $b$ minimal. The parameter $m \cdot d$ was calculated from $a$ with:

$m \cdot d=\frac{a}{g \cdot \sin \left(\varphi_{\text {reclined }}\right)}$

\section{Appendix B}

In order to calculate net muscular torques $\left(T_{\text {musc }}\right)$ from the acceleration data $\left(\ddot{\varphi}_{\mathrm{elb}}\right)$ and the gravitational torque data $\left(T_{\text {grav }}\right)$ with equation,

$T_{\text {musc }}+T_{\text {grav }}=J_{R} \cdot \ddot{\varphi}_{\mathrm{elb}}$

we estimated $J_{R}$, the moment of inertia of the lower arm and manipulandum together relative to the rotational axis of the low-friction hinge $(R)$.

The experimental setup was placed in a $0^{\circ}$ reclined orientation (upright) with the longitudinal axis of the upper arm in line with thorax (see Fig. 7). Participants were asked to keep their lower arm perpendicular to the upper

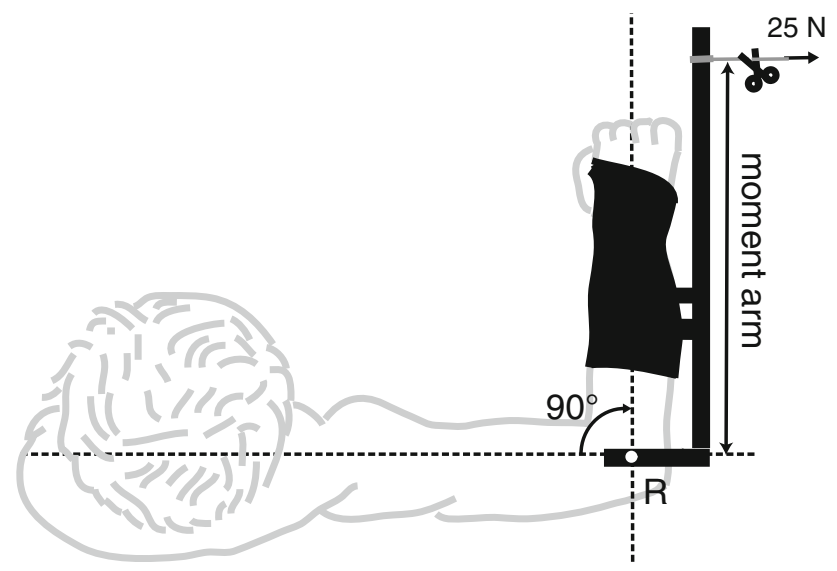

Fig. 7 A top view of the setup used to estimate moment of inertia of lower arm and manipulandum (shown in black) together. The participant is asked to keep the elbow angle in $90^{\circ}$, while a force of $25 \mathrm{~N}$ is applied to the manipulandum by means of a rope. A quickrelease measurement was initiated by unexpectedly cutting the rope arm as a $2.5 \mathrm{~kg}$ mass was attached to the manipulandum at a $0.25-\mathrm{m}$ distance of $\mathrm{R}$ by means of a cord and pulley. Mass was released from the manipulandum by quickly cutting the rope at a randomly chosen time between 0 and $60 \mathrm{~s}$ after the start of the measurement. This quick release of the mass was done out of visual range of the participant to make sure that it came unexpectedly for him/her. For the initial peak in $\ddot{\varphi}_{\text {elb }}$ after the quick release of the mass, we assume that $J_{R} \cdot \ddot{\varphi}_{\text {elb }}$ is equal to the net muscular elbow torque imposed by the mass before it was quickly released.

\section{References}

Bringoux L, Tamura K, Faldon M, Gresty MA, Bronstein AM (2004) Influence of whole-body pitch tilt and kinesthetic cues on the perceived gravity-referenced eye level. Exp Brain Res 155(3):385-392

Carriot J, Dizio P, Nougier V (2008) Vertical frames of reference and control of body orientation. Neurophysiologie Clinique-Clinical Neurophysiol 38(6):423-437

Crevecoeur F, Thonnard JL, Lefevre P (2009) Optimal Integration of gravity in trajectory planning of vertical pointing movements. J Neurophysiol 102(2):786-796. doi:10.1152/jn.00113.2009

Ebenholt SM (1970) Perception of vertical with body Tilt in median plane. J Exp Psychol 83(1):1-6

Elliott D, Hansen S, Mendoza J, Tremblay L (2004) Learning to optimize speed, accuracy, and energy expenditure: a framework for understanding speed-accuracy relations in goal-directed aiming. J Motor Behav 36(3):339-351

Gentili R, Cahouet V, Papaxanthis C (2007) Motor planning of arm movements is direction-dependent in the gravity field. Neuroscience 145(1):20-32

Gribble PL, Ostry DJ (2000) Compensation for loads during arm movements using equilibrium-point control. Exp Brain Res 135(4):474-482

Hansen S, Mendoza J, Tremblay L, Elliott D (2003) Reducing the energy and time expenditures of rapid aiming movements. J Sport Exerc Psychol 25:S65-S65

Kistemaker DA, Wong JD, Gribble PL (2010) The central nervous system does not minimize energy cost in arm movements. J Neurophysiol 104(6):2985-2994

Kurtzer I, DiZio PA, Lackner JR (2005) Adaptation to a novel multiforce environment. Exp Brain Res 164(1):120-132. doi: 10.1007/s00221-005-2216-y

Lackner JR, DiZio P (2000) Human orientation and movement control in weightless and artificial gravity environments. Exp Brain Res 130(1):2-26

Le Seac'h AB, McIntyre J (2007) Multimodal reference frame for the planning of vertical arms movements. Neurosci Lett 423(3): 211-215. doi:10.1016/j.neulet.2007.07.034

Mussa-Ivaldi FA, Hogan N, Bizzi E (1985) Neural, mechanical, and geometric factors subserving arm posture in humans. J Neurosci 5(10):2732-2743

Papaxanthis C, Pozzo T, Stapley P (1998) Effects of movement direction upon kinematic characteristics of vertical arm pointing movements in man. Neurosci Lett 253(2):103-106

Papaxanthis C, Schieppati M, Gentili R, Pozzo T (2002) Imagined and actual arm movements have similar durations when performed under different conditions of direction and mass. Exp Brain Res 143(4):447-452 
Papaxanthis C, Pozzo T, McIntyre J (2005) Kinematic and dynamic processes for the control of pointing movements in humans revealed by short-term exposure to microgravity. Neuroscience 135(2):371-383

Pipereit K, Bock O, Vercher JL (2006) The contribution of proprioceptive feedback to sensorimotor adaptation. Exp Brain Res 174(1):45-52

Scott SH (2004) Optimal feedback control and the neural basis of volitional motor control. Nat Rev Neurosci 5(7):534-546

Smeets JBJ, van den Dobbelsteen JJ, de Grave DDJ, van Beers RJ, Brenner E (2006) Sensory integration does not lead to sensory calibration. Proc Natl Acad Sci USA 103(49):18781-18786
Sober SJ, Sabes PN (2003) Multisensory integration during motor planning. J Neurosci 23(18):6982-6992

Thoroughman KA, Shadmehr R (1999) Electromyographic correlates of learning an internal model of reaching movements. J Neurosci 19(19):8573-8588

Todorov E, Jordan MI (2002) Optimal feedback control as a theory of motor coordination. Nat Neurosci 5(11):1226-1235

Virji-Babul N, Cooke JD, Brown SH (1994) Effects of gravitational forces on single joint arm movements in humans. Exp Brain Res 99(2):338-346 